\title{
Quasi-solid-state dye-sensitized solar cells with siloxane poly(ethylene glycol) hybrid gel electrolyte
}

\author{
James Joseph ${ }^{1}$, Kyung Mo Son ${ }^{2}$, R Vittal ${ }^{2}$, Whasup Lee ${ }^{3}$ \\ and Kang-Jin Kim ${ }^{2,4}$ \\ ${ }^{1}$ Central Electrochemical Research Institute, Karaikudi-630 006, India \\ 2 Division of Chemistry and Molecular Engineering, Korea University, Seoul, 136-713, Korea \\ ${ }^{3}$ Samsung SDI Co. Ltd, Gyeonggi-Do, 449-577, Korea \\ E-mail: kjkim@korea.ac.kr
}

Received 7 October 2005, in final form 14 February 2006

Published 3 April 2006

Online at stacks.iop.org/SST/21/697

\begin{abstract}
A new type of hybrid gel electrolyte matrix based on tetraethyl orthosilicate (TEOS) and poly(ethylene glycol) (Mw 200, PEG $_{200}$ ) was developed for dye-sensitized solar cells (DSSCs). The quasi-solid-state DSSC containing the hybrid gel electrolyte rendered an open-circuit voltage of $0.74 \mathrm{~V}$, a short-circuit photocurrent density of $9.2 \mathrm{~mA} \mathrm{~cm}^{-2}$ and a fill factor of 0.61 at $100 \mathrm{~mW} \mathrm{~cm}^{-2}$, and consequently an overall solar energy conversion efficiency of $4.1 \%$. $\mathrm{TiO}_{2}$ films and gels were characterized by FE-SEM and FT-IR, and the assembled DSSCs by photocurrent density-voltage and incident photon-to-current conversion efficiency measurements.
\end{abstract}

\section{Introduction}

It is well known that the performance of DSSCs is best only when liquid electrolytes are used [1, 2]. However, the commercialization of these cells was impeded owing to technological problems related to hermetic sealing, the precipitation of salts at low temperature and the evaporation of liquids at high temperature.

Replacing liquid electrolytes in DSSCs with solid or quasi-solid electrolytes is expected to make the cells viable. Two of the approaches to replace the liquid electrolytes were the incorporation of hole conducting layers, such as polythiophenes [3], polypyrrole [4, 5], $\mathrm{CuI} / \mathrm{CuSCN}[6,7]$ and of polymer or gelled electrolytes [8-10]. In all these attempts only low overall energy conversion efficiencies were achieved, essentially owing to the imperfect filling of the nanosized pores of the semiconductor film with hole transporting material and the associated inadequate charge mobility in the electrolyte.

Polymer electrolytes have become synonymous with solid state or quasi-solid sate DSSSs, owing to their good ionic conductivities and non-propensity to leakage. In particular, their non-volatile nature is the most decisive

\footnotetext{
4 Author to whom any correspondence should be addressed.
}

property to ensure the long-term stability of the DSSCs. The beneficial effects of in situ photo-polymerized poly[di(ethylene glycol)-2-ethyl hexyl ether acrylate] (PDEA) in the pores of dye-coated $\mathrm{TiO}_{2}$ films on the photovoltaic characteristics of DSSCs were reported recently by our laboratory [11]. Nanosized fumed silica particles were used to gel liquid electrolyte by Grätzel et al [12] and to make a stable poly(ethylene glycol diethyl ether)-based electrolyte by Kim et al [13]. Instead of fumed silica nanoparticles, sol-gel silica/poly(ethylene glycol) nanocomposite thin film was used to support electrolyte; when the nanocomposite film contained considerable amount of residual water, the corresponding solar cell showed low photovoltaic properties and poor stability [14], most likely owing to the adsorption of the residual water within the pores of the $\mathrm{TiO}_{2}$ film and in the nanocomposite gel.

In this paper, we report on the high performance of a quasisolid DSSCs, fabricated by using a novel inorganic-organic hybrid gel from TEOS and $\mathrm{PEG}_{200}$ as the electrolyte matrix. The hybrid gel reported here is essentially a non-aqueous cross-linked polymer and is intended not only for solidifying the electrolyte but also for the suppression of vaporization of solvent molecules and thereby for enhancing the efficiency and stability of the DSSC. 


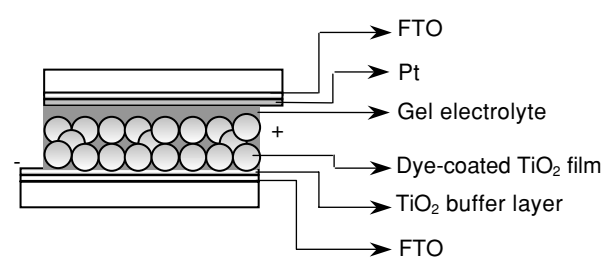

Figure 1. Schematic diagram of the quasi-solid-state DSSC.

\section{Experimental}

A thin non-porous $\mathrm{TiO}_{2}$ buffer layer was deposited on an FTO conducting glass (Libbey-Owen-Ford Co.) by spin coating, using 5\% titanium (IV) butoxide in ethanol, followed by annealing of the $\mathrm{FTO}$ glass at $450{ }^{\circ} \mathrm{C}$. $\mathrm{A} \mathrm{TiO}_{2}$ colloidal mixture was prepared by grinding together in a mortar $1.2 \mathrm{~g}$ of $\mathrm{TiO}_{2}$ (P25 Degussa), $8 \mathrm{ml}$ of water, $0.5 \mathrm{ml}$ of 2,3-pentanedione, $0.24 \mathrm{~g}$ each of poly(ethylene oxide) and poly(ethylene glycol). The paste obtained was then placed in a weighing bottle and stirred well for $24 \mathrm{~h}$. From this colloidal mixture, a $\mathrm{TiO}_{2}$ film of about $10 \mu \mathrm{m}$ thick was made on the above-pretreated FTO glass, using the doctor blade technique. The film was dried and annealed at $450{ }^{\circ} \mathrm{C}$. The $\mathrm{TiO}_{2}$ film thus obtained was impregnated with $0.3 \mathrm{mM}$ of $\left[\mathrm{RuL}_{2}(\mathrm{NCS})_{2}\right] \cdot 2 \mathrm{H}_{2} \mathrm{O}$, $\mathrm{N} 3$ dye (where $\mathrm{L}=2,2^{\prime}$-bipyridine-4, $4^{\prime}$-dicarboxylic acid) in absolute ethanol for $24 \mathrm{~h}$ at room temperature.

The hybrid gel electrolyte was prepared as follows: $3 \mathrm{ml}$ of TEOS, $0.4 \mathrm{ml}$ of glacial acetic acid and $0.5 \mathrm{ml}$ of water were mixed together and sonicated for $15 \mathrm{~min}$. $0.3 \mathrm{ml}$ of $\mathrm{PEG}_{200}\left(d=1.127 \mathrm{~g} \mathrm{~cm}^{-3}\right)$ was then added and sonication continued for 10 more minutes. The amount of water was only $52 \%$ of the stoichiometric value required for the hydrolysis of TEOS. Acetic acid functions as a catalyst in the hydrolysis process. Low molecular weight PEG was intended for both good mechanical strength and good ion transfer [14]. To the contents $7 \mathrm{ml}$ of redox electrolyte, containing $0.05 \mathrm{M}$ iodine, 0.6 M 1-hexyl-2,3-dimethylimidazolium iodide, $0.1 \mathrm{M} \mathrm{LiI}$ and $0.5 \mathrm{M} 4$-tert-butylpyridine in 3-methoxypropionitrile, was added. Thus it is a gelled 3-methoxypropionitrile electrolyte. It is to be noted that both 3-methoxypropionitrile and 4tert-butylpyridine can evaporate. This hybrid gel electrolyte was sonicated for $15 \mathrm{~min}$. It turned viscous in $5 \mathrm{~min}$ on sonication due to the condensation of silanol groups, formed during hydrolysis, with $\mathrm{PEG}_{200}$ in the presence of the basic electrolyte. The above-prepared dye-coated $\mathrm{TiO}_{2}$ film electrode was immersed in the hybrid gel electrolyte for 1 min. Prior to the dip-coating, the back of the FTO was masked with insulating tape in order to prevent contact with the hybrid gel electrolyte. The dip-coated electrode was kept in a clean dish in slanting position and excess solvents were removed from sides using a blotting tissue. The $\mathrm{TiO}_{2}$ electrode coated with the hybrid gel electrolyte was then sandwiched by simple hand pressure onto a Pt-coated FTO counter electrode; the two electrodes were then more firmly pressed using a plastic clip as shown in figure 1. After a few minutes, the siloxane gel bonded the two electrodes, thereby obviating the need to seal the edges of the cell.

FT-IR spectra were obtained using a Braun MB series FTIR spectrometer. For this, samples were prepared by mixing

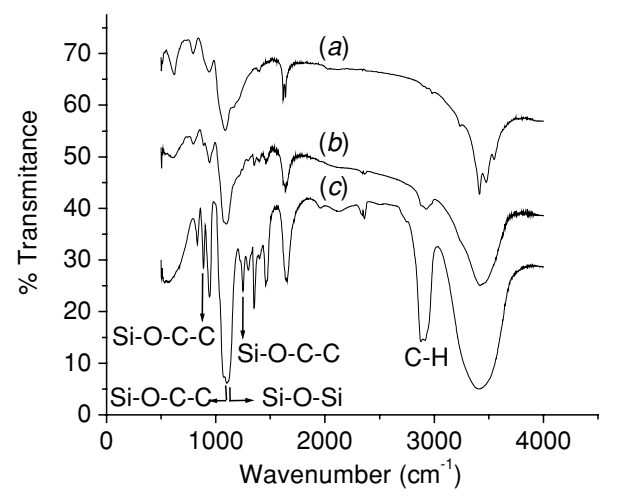

Figure 2. FT-IR spectra of dried gels: (a) gel with bare TEOS, (b) gel with 10:1 of TEOS:PEG and (c) gel with 1:1 of TEOS:PEG.

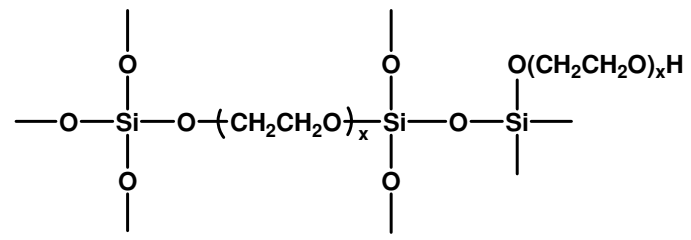

Figure 3. Schematic representation of the structure of the hybrid gel.

$1 \mathrm{ml}$ of TEOS with $0,0.1$ or $1 \mathrm{ml}$ of $\mathrm{PEG}_{200}$, followed by the addition of $0.1 \mathrm{ml}$ of water, $0.1 \mathrm{ml}$ of glacial acetic acid and sonication to a viscous mixture. The mixture was then poured into a clean Petri plate and dried at $50{ }^{\circ} \mathrm{C}$ for $3 \mathrm{~h}$. The homogeneous polymeric solid so obtained was ground with $\mathrm{KBr}$, and pellets were prepared for the analysis. Film morphologies were obtained using a Hitachi S-4300 FE-SEM.

Photocurrent-voltage $(J-V)$ curves of the assembled DSSCs with hybrid gel/liquid electrolytes were obtained using a Keithley Model M236 source measure unit. A $300 \mathrm{~W}$ Xe lamp with an AM 1.5 filter (Oriel) was used to illuminate the DSSCs at the light intensity of 1 sun $\left(100 \mathrm{~mW} \mathrm{~cm}^{-2}\right)$. IPCE values were measured using a photon counting spectrofluorometer (ISS PC1) equipped with a $350 \mathrm{~W}$ Xe lamp and a motorized monochromator. Incident light intensities were calibrated using a photodiode detector (Newport 818UV) and an optical power meter (Newport 1830-C).

\section{Results and discussion}

Curve ' $a$ ' in figure 2 shows the FT-IR spectrum of dried gel with bare TEOS, while curve ' $b$ ' that of gel with 10:1 of TEOS:PEG and curve 'c' that of gel with 1:1 of TEOS:PEG. The spectra show absorption peaks at around $900 \mathrm{~cm}^{-1}$ due to $\mathrm{Si}-\mathrm{O}-\mathrm{C}-\mathrm{C}$ and at $1100 \mathrm{~cm}^{-1}$ due to the overlapping of peaks of $\mathrm{Si}-\mathrm{O}-\mathrm{C}-\mathrm{C}$ and $\mathrm{Si}-\mathrm{O}-\mathrm{Si}$ [15]. The peak at around $2900 \mathrm{~cm}^{-1}$ was assigned to $\mathrm{C}-\mathrm{H}$ stretching, and its increased intensity is due to the increase of $\mathrm{PEG}_{200}$ content. The peak intensity at $3450 \mathrm{~cm}^{-1}$ also increased with increased $\mathrm{PEG}_{200}$ content, indicating an increase in $\mathrm{OH}$ content (from the glycol moiety) [16]. Based on the above observations, the hybrid gel can be represented as shown in figure 3. Figures $4(a)$ and $(b)$ show the top and cross-sectional FE-SEM images of the dye-coated $\mathrm{TiO}_{2}$ film before the impregnation of the hybrid gel electrolyte 

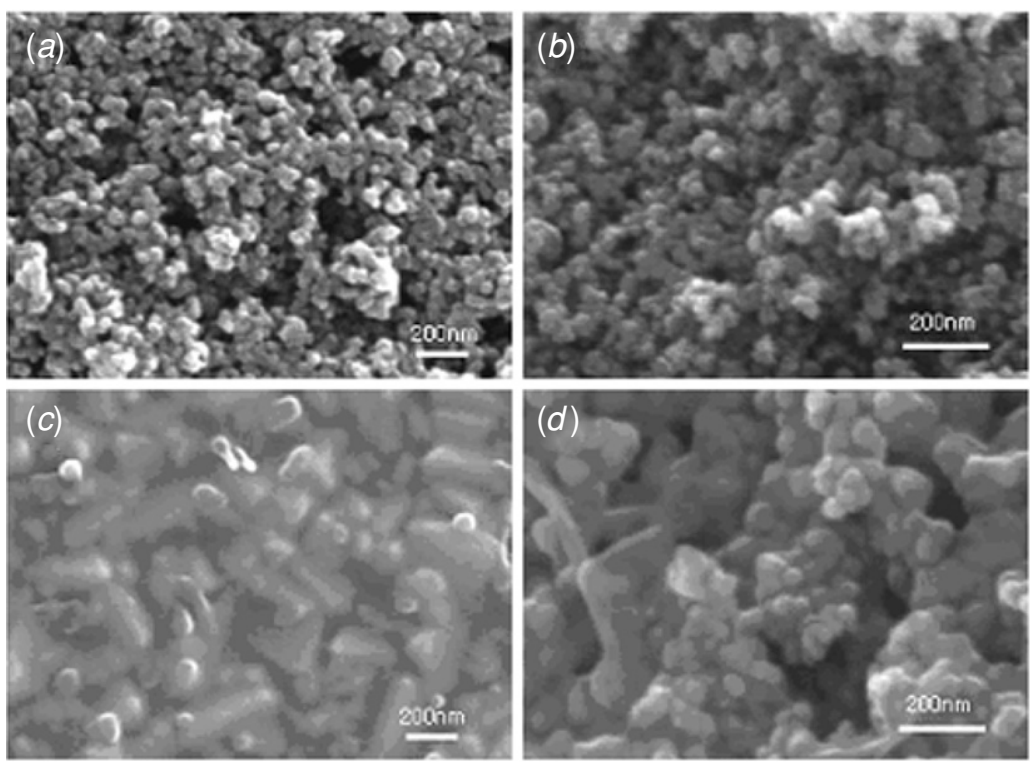

Figure 4. FE-SEM images of a dye-coated $\mathrm{TiO}_{2}$ film: $(a)$ and $(b)$ are the top and cross section before the impregnation of the hybrid gel electrolyte and $(c)$ and $(d)$ are their respective images after gel impregnation. The cross section images were recorded at a depth of $5 \mu \mathrm{m}$ from the top surface of the film.

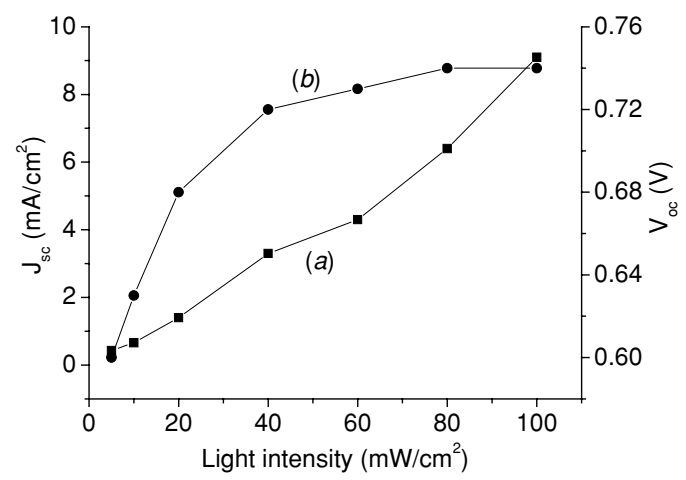

Figure 5. Dependence of $(a)$ the short-circuit photocurrent and (b) open-circuit voltage of a DSSC with hybrid gel electrolyte on the light intensity.

and figures $4(c)$ and $(d)$ their respective corresponding images after the impregnation. Before the impregnation of the hybrid gel electrolyte, the $\mathrm{TiO}_{2}$ nanoparticles are distinguishable and after the impregnation they are covered with the gel electrolyte, indicating good contact between the $\mathrm{TiO}_{2}$ particles and the electrolyte. These images clearly indicate that the hybrid gel electrolyte penetrates deep into the pores of the $\mathrm{TiO}_{2}$ film (figure $4(d)$ ) and is uniformly distributed in the film. This penetration of electrolyte is essential for interfacial contact between it and the dye-coated $\mathrm{TiO}_{2}$ film.

Figure 5 shows the dependence of short-circuit photocurrent density $\left(J_{\mathrm{sc}}\right)$ and open-circuit voltage $\left(V_{\mathrm{oc}}\right)$ of the DSSC containing the hybrid gel electrolyte on the light intensity up to $100 \mathrm{~mW} \mathrm{~cm} \mathrm{~cm}^{-2}$. A near linear relationship between $J_{\mathrm{sc}}$ and intensity of light is noted. This linearity implies that the photocurrent is neither limited by the diffusion of $\mathrm{I}_{3}^{-} / \mathrm{I}^{-}$and the cations in the network structure of TEOS$\mathrm{PEG}_{200}$-based hybrid gel nor by their migration. The reasonably good conductivity of the electrolyte for the redox species rationalizes the linearity. It suggests that the threedimensional network structure of the hybrid gel, with its additional flexibility owing to the $\mathrm{PEG}_{200}$, can retain the electrolyte in its pores, and the electrolyte can percolate freely, thereby giving the hybrid gel good ionic conductivity. On the other hand, the $V_{\text {oc }}$ increases logarithmically with the increase of light intensity, in accordance with the relationship [2]

$$
V_{\mathrm{oc}}=(k T / e) \ln \left(J_{\mathrm{sc}} / J_{\mathrm{o}}\right)
$$

where $J_{0}$ is the exchange current density. Similar intensity-dependent $J-V$ curves have been obtained by Nogueira et al with polymer electrolytes employing the copolymer, poly(epichlorohydrin-co-ethylene oxide), Epichlomer-16 [17].

Maximum solar energy conversion efficiency was obtained for the hybrid gel containing TEOS: $\mathrm{PEG}_{200}$ at a ratio of 10:1 by volume. All the results reported here regarding the photovoltaic performance of quasi-solid-state DSSC are of that cell containing hybrid gel of this composition. Curve 'a' in figure 6 represents the typical $J-V$ behaviour of a DSSC using the hybrid gel electrolyte at an illumination intensity of $100 \mathrm{~mW} \mathrm{~cm} \mathrm{~m}^{-2}$. With the $V_{\mathrm{oc}}$ of $0.74 \mathrm{~V}$, the $J_{\mathrm{sc}}$ of $9.2 \mathrm{~mA} \mathrm{~cm}^{-2}$ and the fill factor of 0.61 , an overall solar energy conversion efficiency of $4.1 \%$ was achieved. The performance of the DSSC is far better than that of the cell fabricated through similar approach by Stathatos et al, i.e., they obtained an opencircuit voltage of $0.45 \mathrm{~V}$, a short-circuit photocurrent density of $4.3 \mathrm{~mA} \mathrm{~cm}^{-2}$ and a fill factor of 0.51 at $96 \mathrm{~mW} \mathrm{~cm} \mathrm{~cm}^{-2}$ [14]. The discrepancy appears to arise from the difference in contents of the electrolyte. In particular, their cell contained some adsorbed water; the presence of residual water in the $\mathrm{TiO}_{2}$ pores and in the nanocomposite gel apparently resulted in the low photovoltaic performance of their cell. In contrast, the water added to the gelation mixture in this study was only $52 \%$ of that required for the hydrolysis of TEOS. Traces of water present in the electrolyte would react with the excess TEOS, making our cell completely free of water. 


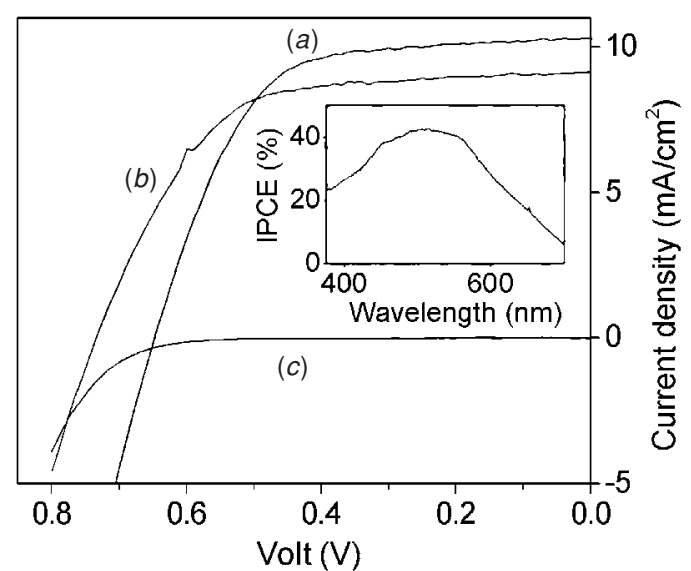

Figure 6. $J-V$ curves of DSSCs with (a) quasi-solid electrolyte and (b) liquid electrolyte at a light intensity of $100 \mathrm{~mW} \mathrm{~cm}^{-2}$. The IPCE spectrum and dark current-voltage curve of the quasi-solid-state DSSC are shown in the inset and by curve $(c)$, respectively.

Curve ' $b$ ' in figure 6 shows the $J-V$ behaviour of a DSSC with a liquid electrolyte with regular 3-methoxypropionitrile solvent and fabricated under the same conditions as those used for a gel electrolyte DSSC. Compared with the case of the DSSC using the gel electrolyte, the $J_{\text {sc }}$ has enhanced by $1.1 \mathrm{~mA} \mathrm{~cm}{ }^{-2}$ and the $V_{\text {oc }}$ by $90 \mathrm{mV}$. The high $V_{\text {oc }}$ value $(0.74 \mathrm{~V})$ of the DSSC with a quasi-solid electrolyte is comparable to the best values obtained by others, using similar quasi-solid-state electrolytes $[8,12,18,19]$. This $V_{\text {oc }}$ value kinetically manifests a low degree of back electron transfer from the $\mathrm{TiO}_{2}$ conduction band to the $\mathrm{I}_{3}^{-}$in the electrolyte (equation (1)) [2, 20]. The TEOS-PEG 200 hybrid gel of the cell apparently retards the back electron transfer rate, because of the reduction in the deep surface states of $\mathrm{TiO}_{2}$ owing to the interaction between the ethylene moieties of $\mathrm{PEG}_{200}$ and the hydrophilic dye-coated $\mathrm{TiO}_{2}$ surface $[10,18]$. Another explanation for high $V_{\mathrm{oc}}$ is the suppression of the back electron transfer by the polymer existing on the surface of $\mathrm{TiO}_{2}$ particles. In energetics, high $V_{\mathrm{oc}}$ indicates a shifting of the conduction band edge of $\mathrm{TiO}_{2}$ towards a more negative value. The base, 4-tert-butylpyridine is known to shift the conduction band edge to a slightly negative potential $[2,21]$. Additionally, the oxygen atoms in the ethylene glycol moieties are also known to shift the conduction band negatively, owing to their Lewis base nature $[17,18]$, thereby increasing the $V_{\text {oc }}$, over that of a DSSC fabricated without using a hybrid gel electrolyte.

The $J_{\text {sc }}$ value of $9.2 \mathrm{~mA} \mathrm{~cm}^{-2}$ (curve 'a' in figure 6) is as high as the values obtained in the case of DSSCs with electrolytes containing fumed silica nanoparticles [12, 13], but better than the one obtained in the case of a DSSC with tetramethoxysilane-produced silica and PEG as electrolyte support [14]. $\mathrm{I}^{-}$and $\mathrm{I}_{3}^{-}$diffusion coefficients in different gel electrolytes were measured by other authors previously $[12,22]$. Though the gelling method is different, the diffusion coefficient values were found to be nearly the same for gel electrolytes and blank liquid electrolytes. These two references suggest that diffusion coefficients may not play a decisive role in the behaviour of $J_{\mathrm{sc}}$ and there are other factors to be considered. The better than expected diffusion coefficient in the case of the gel electrolyte can be partially explained by the fact that 1-hexyl-2,3-dimethylimidazolium iodide, being a liquid electrolyte, itself can facilitate the formation of liquid cannels through which the diffusion of $\mathrm{I}^{-}$and $\mathrm{I}_{3}^{-}$become facile, which in turn renders higher $J_{\mathrm{sc}}$ than expected for such a gel electrolyte cell. In the case of the liquid electrolyte DSSC, with the $V_{\mathrm{oc}}$ of $0.65 \mathrm{~V}$, the $J_{\mathrm{sc}}$ of $10.3 \mathrm{~mA} \mathrm{~cm}^{-2}$ and the fill factor of 0.62 , an overall solar energy conversion efficiency of $4.2 \%$ was achieved. Contact between dye molecules and gel electrolyte, necessary for the regeneration of dye, may not be as perfect as that in the case of dye molecules and liquid electrolyte. This can be a reason for the slightly lesser $J_{\text {sc }}$ observed in the case of the gel electrolyte relative to that in the case of the liquid electrolyte (figure 6). Given the quasi-solid-state nature of the electrolyte it is notable that its pertinent DSSC has an overall energy conversion efficiency comparable to that of a cell with organic liquid electrolyte. The beneficial effects of 4-tert-butylpyridine and 1-hexyl-2,3-dimethylimidazolium iodide in terms of the enhancement of current density were well documented [12, 23].

The photocurrent action spectrum (inset in figure 6) shows that the maximum IPCE value of $43 \%$ at $525 \mathrm{~nm}$ is slightly lower than those reported in similar studies, but our IPCE values showed a smooth decrease in both longer and shorter wavelength regions with respect to the maximum value, compared with the steep decrease at longer and shorter wavelength regions (with respect to the maximum value) in similar investigations [12-14]. This broadened IPCE spectrum might be the result of increased light scattering from large aggregates of hybrid-gel-covered $\mathrm{TiO}_{2}$ particles, as can be understood from the FE-SEM images of the hybrid-gel-coated $\mathrm{TiO}_{2}$ electrode (figures $4(c)$ and $(d)$ ). The maximum IPCE peak at $525 \mathrm{~nm}$ owing to the absorption of the Ru sensitizer also implies that detrimental effect to $J_{\text {sc }}$ due to light absorption of the gelator is negligible in the present conditions, which also explains the high $J_{\text {sc }}$ obtained with the gel electrolyte cell.

High $J_{\mathrm{sc}}$ and $V_{\mathrm{oc}}$ with moderately high solar conversion efficiency make this electrolyte attractive for quasi-solid-state DSSCs. We assume that the stoichiometrically lesser water used for the preparation of our gel has facilitated slower rates of hydrolysis and condensation during its formation to produce a gel having close contact and compatibility with $\mathrm{TiO}_{2}$ and enough spaces for the movement of redox species. This is reflected by the high $V_{\mathrm{oc}}, J_{\mathrm{sc}}$ and linearity between $J_{\mathrm{sc}}$ and illumination intensity obtained as above. Furthermore, waterfree hybrid gel is advantageous for increasing the stability of the DSSCs, as gaseous products from water decomposition as a result of absorption of UV light by $\mathrm{TiO}_{2}$ can be avoided. We have not carried out stability tests for the DSSCs because the cells were not sealed. However, a few preliminary experiments with the unsealed cells showed about $30 \%$ reduction in $J_{\mathrm{sc}}$ values, but the $V_{\mathrm{oc}}$ remained the same, after $48 \mathrm{~h}$ of assembling the cells. Drying of the hybrid gel is probably the reason for the reduction in the current density. Optimization of hybrid gel parameters for its good contact with the dye-coated $\mathrm{TiO}_{2}$ surface and for increasing ionic conductivity, and of cell assembly with proper sealing, will certainly enhance the solar energy conversion efficiency and the stability of the DCCS to much higher degree. 


\section{Conclusion}

A dye-sensitized solar cell was constructed by preparing a novel tetraethyl orthosilicate-poly(ethylene glycol) hybrid gel as the electrolyte matrix for the redox couple, $\mathrm{I}_{3}^{-} / \mathrm{I}^{-}$. FTIR spectra indicated the formation of the hybrid gel. FESEM images showed that the viscous hybrid gel electrolyte complex penetrated deep into the pores of dye-coated $\mathrm{TiO}_{2}$ film and covered well the $\mathrm{TiO}_{2}$ particles. Analysis of the dependence of photovoltaic properties of the cell on the light intensity indicated that the conductivity of the electrolyte was reasonably good. The new DSSC showed $J_{\mathrm{sc}}, V_{\mathrm{oc}}$ and an overall solar energy conversion efficiency, comparable to those obtained with similar quasi-solid DSSCs developed by other researchers. The interaction between the hydrophilic $\mathrm{TiO}_{2}$ surface and the siloxane-PEG ${ }_{200}$ hybrid gel with Lewis base nature, at the $\mathrm{TiO}_{2} /$ electrolyte interface, is considered to be the reason for the reduction in the back electron transfer from the $\mathrm{TiO}_{2}$ conduction band to the $\mathrm{I}_{3}^{-}$in the hybrid gel electrolyte and thereby for the high $V_{\mathrm{oc}}$.

\section{Acknowledgments}

This work was supported by CRM-KOSEF of Korea University and by the Sol-Gel Innovation Project.

\section{References}

[1] Grätzel M 2004 J. Photochem. Photobiol. A: Chem. 3 164

[2] Sze S M 1981 Physics of Semiconductor Devices (New York: Wiley) p 264

[3] Smestad G P, Spiekermann S, Kowalik J, Grant C D, Schwartzberg A M, Zhang J, Tolbert L M and Moons E 2003 Sol. Energy Mater. Sol. Cells 7685
[4] Saito Y, Fukuri N, Senadeera R, Kitamura T, Wada Y and Yanagida S 2004 Electrochem. Commum. 671

[5] Kitamura T, Maitani M, Wada Y and Yanagida S 2001 Chem. Lett. 91054

[6] Meng Q B, Takahashi K, Zhang X T, Sutanto I, Rao T N, Sato O, Fujishima A, Watanabe H, Nakamori T and Uragami M 2003 Langmuir 193572

[7] Regan B O, Lenzmann F, Muis R and Wenke J 2002 Chem. Mater. 145023

[8] Kubo W, Kitamura T, Hanabusa T, Wada Y and Yanagida S 2002 Chem. Commun. 4374

[9] Matsumoto M, Wada Y, Kitamura T, Shigaki K, Inoue T, Ikeda M and Yanagida S 2001 Bull. Chem. Soc. Japan 74387

[10] Stergiopoulos T, Ioannis M A, Arabatzis M, Georgios K and Polycarpos F 2002 Nano Lett. 21259

[11] Kal S H, Joseph J, Lee J and Kim K J 2005 J. Electrochem. Soc. 152 A1378

[12] Wang P, Zakeeruddin S M, Comte P, Exnar I and Grätzel M $2003 \mathrm{~J}$. Am. Chem. Soc. 1251166

[13] Kim J H, Kang M S, Kim Y J, Won J, Park N G and Kang Y S 2004 Chem. Commun. 141662

[14] Stathatos E, Lianos P and Krontiras C 2001 J. Phys. Chem. B 1053486

[15] Silverstein R M, Bassler G C and Morrill T C 1991 Spectrometric Identification of Organic Compounds 5 th edn (New York: Wiley) p 162

[16] Orel B, Krasovec U O and Stangar U L 1998 J. Sol-Gel Sci. Technol. 1187

[17] Nogueira A F, Durrant J R and De Paoli M A 2001 Adv. Mater. 13826

[18] Komiya R, Han L, Yamanaka R, Islam A and Mitate T 2004 J. Photochem. Photobiol. A: Chem. 164123

[19] Stathatos E, Lianos P, Vuk A S and Orel B 2004 Adv. Funct. Mater. 1445

[20] Kaven L and Grtäzel M 1989 Electrochim. Acta 341327

[21] Boschloo G, Lindstrom H, Magnusson E, Holmberg A and Hagfeldt A 2002 J Photochem. Photobiol. A: Chem. 14811

[22] Wang P, Zakeeruddin S M, Exnar I and Grätzel M 2002 Chem. Commun. 242972

[23] Huang S Y, Schlichthörl G, Nozik A J, Grätzel M and Frank A J 1997 J. Phys. Chem. B 101257 